\title{
WEYL'S LEMMA FOR POINTWISE SOLUTIONS OF ELLIPTIC EQUATIONS
}

\author{
J. R. DIEDERICH
}

\begin{abstract}
We prove that pointwise, $L_{1}$ solutions of second
\end{abstract} order elliptic partial differential equations are classical solutions.

0. Introduction. A consequence of Weyl's lemma for second order elliptic partial differential equations is that every $L_{1}$ (Lebesgue class) weak solution is a classical solution, i.e., if, for

$$
L=\sum_{i, j=1}^{K} a_{i j}(x) \partial^{2} / \partial x_{i} \partial x_{j}+\sum_{i=1}^{K} b_{i}(x) \partial / \partial x_{i}+c(x)
$$

and

$$
L^{*}=\sum_{i, j=1}^{K} \partial^{2} / \partial x_{i} \partial x_{j} a_{i j}(x)-\sum_{i=1}^{K} \partial / \partial x_{i} b_{i}(x)+c(x),
$$

we have $\int_{\Omega} u L^{*} \phi=\int_{\Omega} f \phi$ for all $\phi$ in $C_{c}^{\infty}(\Omega)$, then $L u=f$ in $\Omega$.

The differentiability of the coefficients of $L$, required for the definition of $L^{*}$, is not intrinsic in view of the maximum principle and the Dirichlet problem (in the case $c(x) \leqq 0$ ). Our aim is to employ a notion of generalized solution of $L u=f$ which bypasses the adjoint operator and thereby establish an analogue of Weyl's lemma when the coefficients are in a Hölder- $\alpha$ class. Definitions and the statement of the theorem follow in the next section.

1. Preliminaries. We shall work in $K$-dimensional Euclidean space, $R^{K}, 3 \leqq K$, and shall use the following notation: $x=\left(x_{1}, \cdots, x_{K}\right)$ and $B(x, r)=$ the open $K$-ball centered at $x$ with radius $r$. $\Omega$ will denote an open set in $R^{K} ;|E|$, the Lebesgue measure of $E ; \partial E$, the boundary of $E$; subscripted $e$, constants which depend only on the operator (1) and $K$; and $\omega_{K}$, the surface area of $\partial B(0,1)$.

A function $u$, which is in $L_{1}$ in a neighborhood of $x$, is said to be in

Presented to the Society, April 22, 1972; received by the editors December 3, 1971 and, in revised form, May 5, 1972.

AMS (MOS) subject classifications (1969). Primary 3542; Secondary 3524.

Key words and phrases. Second order elliptic equations, generalized partials, pointwise solution, regularity.

American Mathematical Society 1973 
$\boldsymbol{t}_{\beta}(x)$ if there is a polynomial $P(y)$ of degree $\leqq \beta$ such that

$$
r^{-K} \int_{B(0 . r)}|u(x+y)-P(y)| d y=o\left(r^{\beta}\right) \quad \text { as } r \rightarrow 0 .
$$

For $\beta=2, P(y)=\gamma_{0}+\sum_{i=1}^{K} \gamma_{i} y_{i}+2^{-1} \sum_{i, j=1}^{K} \gamma_{i j} y_{i} y_{j}$, where $\gamma_{i j}=\gamma_{j i}$; we define the generalized partial derivatives $D_{i} u(x), D_{i j} u(x)$ to be $\gamma_{i}, \gamma_{i j}$ respectively. Also we can redefine $u$ at $x$, if necessary, so that $u(x)=\gamma_{0}$. (The class $t_{\beta}$ was defined in [1] and has since appeared in [2] and [6].)

For $u$ in $t_{2}(x)$, we say that $u$ is a $t_{2}$ solution of $L u=f$ at $x$ if

$$
\sum_{i, j=1}^{K} a_{i j}(x) D_{i j} u(x)+\sum_{i=1}^{K} b_{i}(x) D_{i} u(x)+c(x) u(x)=f(x) \text {. }
$$

We say that $u$ is a $t_{2}$ solution of $L u=f$ in $\Omega$ if $u$ is a $t_{2}$ solution at each point in $\Omega . u$ is said to be a classical solution if $u \in C^{2}(\Omega)$. (It should be noted that no restrictions have been placed on the operator $L$ up to this point.)

We shall assume the operator (1) to be elliptic and have $\alpha$-Hölder continuous coefficients, $0<\alpha \leqq 1$; with this, (1) is uniformly elliptic on compact subsets of $\Omega$.

THEOREM. If $u$ is an $L_{1}, t_{2}$ solution of $L u=f$ in $\Omega$, then $u$ is a classical solution.

REMARKS. No assumption has been made on the integrability of the generalized derivatives $D_{i} u(x), D_{i j} u(x)$ when considered as functions on $\Omega$. In fact, see [2], there are simple examples of functions in $t_{2}(x), x$ in $(-1,1)$ such that $D_{1} u(x), D_{11} u(x)$ are not in $L_{1}$ on compact subsets of $(-1,1)$; note that the $o\left(r^{\beta}\right)$ in (2) is not assumed to be uniform in $x$.

The special case of $u$ in $L_{\infty}, f \equiv 0$, and $c(x) \leqq 0$, has been established in [2]. The case for general $f, \alpha$-Hölder continuous, is immediate. The restriction on $c(x)$ is not essential and while a nontrivial argument is necessary for unrestricted $c(x)$, the techniques are essentially those of [2, $\S \S 1-4]$, and those of this paper. We therefore maintain $c(x) \leqq 0$.

As in [2], we only need assume that $u$ is a $t_{2}$ solution of $L u=f$ almost everywhere and in $t_{2}$ everywhere in $\Omega$. We shall omit this extension.

It is not clear that the Hölder- $\alpha$ condition is best possible. However, our result is best possible with respect to the notion of $t_{2}$ solution in that the conclusion fails if the absolute values in (2) are not required, i.e., $u(x)=x_{1}|x|^{-K}$ satisfies, with $P(y) \equiv 0$ for $x=0$,

$$
r^{-K} \int_{B(0, r)} u(x+y)-P(y) d y=o\left(r^{2}\right) \quad \text { as } r \rightarrow 0
$$

for all $x$; that is, $x_{1}|x|^{-K}$ has generalized Laplacian equal to zero everywhere and is clearly not harmonic at 0 . 
Our theorem combined with [2] allows for an immediate generalization of Serrin's Theorem 1 [4] with respect to removable $F_{\sigma}$ sets.

A forthcoming paper in the Ann. Scuola Norm. Sup. Pisa by Hager and Ross deals with elliptic equations in divergence form with $\alpha$-Hölder coefficients; the notion of weak solution is considered vis-à-vis the notion of pointwise solution employed in this paper.

This work is an extension of some of the concepts developed by V. L. Shapiro in [6].

We also wish to thank the referee for his many helpful suggestions.

2. Fundamental lemmas. The first three lemmas were established in [2]. By a portion $P$ of a set $Z$, we shall mean a nonempty intersection of $Z$ with an open ball.

LeMma 1 [2, Lemma 2]. If $u$ is in $t_{2}(x)$ for all $x$ in $Z$ and if $Z$ is closed and nonempty, then there is a portion $P$ of $Z$ such that $u$ is continuous in $P$ in the relative topology, i.e., if $x_{1} \in P$, then given $\varepsilon>0$, there is a $\delta>0$ such that for $x_{2} \in P$ and $\left|x_{1}-x_{2}\right|<\delta,\left|u\left(x_{1}\right)-u\left(x_{2}\right)\right|<\varepsilon$.

LEMMA 2 (SEE [2, PROOF OF THEOREM 1]). If $u$ is in $t_{\mathbf{2}}(x)$ for all $x$ in $Z$, closed and nonempty, then there is a portion $P$ of $Z$ and positive constants $M$ and $r_{0}$ such that for all $x$ in $P$ and $0<r<r_{0}$,

$$
|B(0, r)|^{-1} \int_{B(0 . r)}|u(x+y)-u(x)| d y \leqq M .
$$

LEMMA 3 [2, THEOREM 1]. If $u$ is an $L_{x}, t_{2}$ solution of $L u=0(c(x) \leqq 0)$ in $\Omega$, then $u$ is a classical solution.

LEMMA 4 (J. SERRIN, [3, p. 300]). There exist functions $K_{+}(x, y)$ and $K_{-}(x, y)$ for $|x| \leqq r,|y|=r, x \neq y$, and $r \leqq 1$ having the following properties:

(i) Considered as functions of $x$ for fixed $y$,

$$
L K_{+} \geqq 0 \text { and } L K_{-} \leqq 0 .
$$

(ii) For any point $y_{0},\left|y_{0}\right|=r$, and any continuous function $g(y)$,

$$
\lim _{x \rightarrow y_{0}} \int_{\partial B(0, r)} K_{ \pm}(x, y) g(y) d S_{r}(y)=g\left(y_{0}\right)
$$

where $d S_{r}(y)$ is the natural surface area element.

(iii) There are positive constants $e_{1}$ and $e_{2}$ such that, for $|x| \leqq r / 3$,

$$
K_{+}(x, y) \geqq e_{1} r^{1-K} \text { and } K_{-}(x, y) \leqq e_{2} r^{1-K} .
$$

Lemma 5. If $L u=0$ in $B\left(x_{0}, r\right)$, then

$$
\left|u\left(x_{0}\right)\right| \leqq e_{3}\left|B\left(x_{0}, r\right)\right|^{-1} \int_{B\left(x_{0}, r\right)}|u(x)| d x .
$$


Proof. As in [4], $u(x)=\int_{\partial J_{3}\left(x_{0}, \rho\right)} u(y) d \omega(x, y)$ where $d(1)(x, y)$ is a nonnegative Borel measure on $\partial B\left(x_{0}, \rho\right)$, with $\rho<r$. Thus

$$
|u(x)| \leqq \int_{\partial B\left(x_{0}, \rho\right)}|u(y)| d \omega(x, y) .
$$

Form

$$
u_{-}(x)=\int_{\partial B\left(x_{0}, \rho\right)} K_{-}(x, y)|u(y)| d S_{\rho}(y) .
$$

By (5), (6), and the maximum principle,

$$
\int_{\partial B\left(x_{0}, \rho\right)}|u(y)| d \omega(x, y) \leqq u_{-}(x) \text { for } x \text { in } B\left(x_{0}, \rho\right),
$$

and by (7),

$$
u_{-}(x) \leqq e_{2} \rho^{1-K} \int_{\partial J\left(x_{0}, \rho\right)}|u(y)| d S_{\rho}(y) \quad \text { for }\left|x-x_{0}\right| \leqq \rho / 3 .
$$

Hence

$$
\left|u\left(x_{0}\right)\right| \leqq e_{2} \rho^{1-K} \int_{\partial B\left(x_{0}, \rho\right)}|u(y)| d S_{\rho}(y) .
$$

Thus by integrating

which yields (8).

$$
\frac{r^{K}}{K}\left|u\left(x_{0}\right)\right| \leqq e_{2} \int_{B\left(x_{0} . r\right)}|u(y)| d y
$$

ProOF OF THE THEOREM $(c(x) \leqq 0)$. Let $Z$ be the set of discontinuities of $u ; Z$ is its closure. If $Z$ is empty, then by Lemma $3, u$ is a classical solution. Assuming, therefore, that $Z \neq \varnothing$, we have, by Lemmas 1 and 2 , a portion $P$ of $Z$ in which $u$ is continuous in the relative topology and satisfies (4) for $x$ in $P$. Let $x_{0} \in P$. If we can show that there is a $\delta>0$ such that $u$ is bounded in $\left|x-x_{0}\right|<\delta$, then $u$ is a classical solution in $\left|x-x_{0}\right|<\delta$. Consequently $x_{0}$ is not in $Z$; therefore $x_{0}$ is not in $P$, which is a contradiction based on the assumption that $Z \neq \varnothing$.

We can choose $\delta=\min \left(r_{0} / 2, r_{1} / 2\right)$, where $r_{0}$ is given in Lemma 2 and where $r_{1}$ is selected so that

(i) $0<r_{1}$,

(ii) $\left\{\left|x_{0}-x\right|<r_{1}, x\right.$ in $\left.Z,\left|u(x)-u\left(x_{0}\right)\right|<1\right\} \subseteq P$.

For $\left|x-x_{0}\right|<\delta, x$ in $Z$,

$$
|u(x)| \leqq\left|u(x)-u\left(x_{0}\right)\right|+\left|u\left(x_{0}\right)\right| \leqq\left|u\left(x_{0}\right)\right|+1 .
$$

For $\left|x-x_{0}\right|<\delta, x$ not in $Z$, there is a point $x^{*}$ in $P$ and $0<\rho<\delta$ such that $x^{*} \in \partial B(x, \rho)$ while $P \cap B(x, \rho)=\varnothing$. Thus $L u=0$ in $B(x, \rho)$. Hence, 
by (8),

$$
\begin{aligned}
|u(x)| & \leqq e_{3}|B(x, \rho)|^{-1} \int_{B(x, \rho)}|u(y)| d y \\
& \leqq e_{3} 2^{K}\left|B\left(x^{*}, 2 \rho\right)\right|^{-1} \int_{B\left(x^{*}, 2 \rho\right)}|u(y)| d y .
\end{aligned}
$$

Since $\rho<\delta<r_{0} / 2,2 \rho<r_{0}$, and $x^{*} \in P$,

$$
\leqq e_{3} 2^{K}\left[\left|u\left(x^{*}\right)\right|+M\right] \text {. }
$$

Since $\left|x_{0}-x^{*}\right| \leqq\left|x_{0}-x\right|+\left|x-x^{*}\right| \leqq \delta+\rho<r_{1},\left|u\left(x^{*}\right)\right| \leqq\left|u\left(x_{0}\right)\right|+1$, which gives $|u(x)| \leqq e_{3} 2^{K}\left[\left|u\left(x_{0}\right)\right|+M+1\right]$, completing the proof.

\section{BIBLIOGRAPHY}

1. A. P. Calderón and A. Zygmund, Local properties of solutions of elliptic partial differential equations, Studia Math. 20 (1961), 171-225. MR 25 \#310.

2. J. R. Diederich, Removable sets for pointwise solutions of elliptic partial differential equations, Trans. Amer. Math. Soc. 165 (1972), 333-352.

3. J. B. Serrin, Jr., On the Harnack inequality for linear elliptic equations, J. Analyse Math. 4 (1955/56), 292-308. MR 18, 398.

4. - Removable singularities of solutions of elliptic equations, Arch. Rational Mech. Anal. 17 (1964), 67-78. MR 30 \#336.

5. V. L. Shapiro, Fourier series in several variables, Bull. Amer. Math. Soc. 70 (1964), 48-93. MR 28 \#1448.

6. - Removable sets for pointwise solutions of the generalized Cauchy-Riemann equations, Ann. of Math. (2) 92 (1970), 82-101.

Department of Mathematics, University of California, Davis, California 95616 